\title{
Microneedling with injectable platelet-rich fibrin for facial rejuvenation
}

\author{
Anna-Maria Vesala ${ }^{1,2}$, Cleopatra Nacopoulos ${ }^{1,3}$, Kalliopi Gkouskou ${ }^{3}$, Francesco Amenta ${ }^{2}$, Emanuele Ruga ${ }^{2}$ \\ ${ }^{1}$ Facial Aesthetics and Dental Clinic, Athens 11523 , Greece. \\ ${ }^{2}$ Clinical Research, Telemedicine and TelePharmacy Centre, School of Medicinal and Health Products Sciences, University of Cameri \\ no, Camerino 62032, Italy. \\ ${ }^{3}$ Laboratory of Research for the Musculoskeletal System (LRMS), School of Medicine, University of Athens, Kifisia 14561, Greece.
}

Correspondence to: Dr. Anna-Maria Vesala, Facial aesthetics and Dental Clinic, 103, Kifisias Ave, Athens 11523, Greece. E-mail vesala.am@gmail.com

How to cite this article: Vesala AM, Nacopoulos C, Gkouskou K, Amenta F, Ruga E. Microneedling with injectable platelet-rich fibrin for facial rejuvenation. Plast Aesthet Res 2021;8:53. https://dx.doi.org/10.20517/2347-9264.2021.57

Received: 2 Jun 2021 First Decision: 23 Jun 2021 Revised: 1 Jul 2021 Accepted: 12 Aug 2021 Published: 19 Sep 2021

Academic Editors: Wen-Guo Cui, Pietro Gentile Copy Editor: Xi-Jun Chen Production Editor: Xi-Jun Chen

\begin{abstract}
Aim: The aim of this literature review is to evaluate the efficacy of microneedling treatment with injectable plateletrich fibrin (i-PRF) for facial skin rejuvenation applications, using an objective skin analysis system and validated patient-reported outcome measures.

Methods: The search approach involved the exploration of electronic databases. An advanced search option was applied to filter our search line, i.e., from February 2011 to April 2021. We performed a search on Medline, Scopus, Embase, and Web of Science, while improving the accessed articles via Ovid interface. Our keywords were chiefly aligned with a combination of MeSH terms and text words. All retrieved articles were written in English.

Results: The search yielded 73 studies. After reviewing their title and summary, nine of them were found to meet the inclusion criteria and, next, the full-text articles were reviewed. Of these, three studies were excluded from systematic research, as they would no longer meet the inclusion criteria. In total, six studies were considered for review.
\end{abstract}

Conclusion: Microneedling treatments combined with blood concentrates are increasingly being utilized as autologous products for aesthetic purposes. Few works can be found on i-PRF in facial rejuvenation, and even fewer on i-PRF along with microneedling. Combined applications seem to be promising and minimally invasive. Further research on PRP and PRF is warranted to better elucidate their functional roles in medical cosmetic rejuvenation. 
Keywords: Microneedling, injectable platelet-rich fibrin, autologous, mesotherapy, skin rejuvenation

\section{INTRODUCTION}

Skin aging signs and symptoms echo the collaborative effects of gravity, progressive bone resorption, decreased tissue elasticity, and redistribution of subcutaneous fullness ${ }^{[1]}$. Skin needling, also called microneedling therapy or collagen induction therapy, is a minimally invasive non-surgical and non-ablative facial rejuvenation therapy involving monitored skin injury induced by a medical microneedling device ${ }^{[2]}$. Wrinkled skin areas that can greatly benefit from microneedling therapy include the areas around the eyes (periocular) and the lips (perilabial), the cheeks, the neck, and the décolleté ${ }^{[2]}$. Such therapies can also prove beneficial in other body areas, e.g., the back of the hands and the arms. Skin needling involves the penetration of variously sized needles (according to individualized patient treatment plans) into the skin in order to rejuvenate affected skin areas ${ }^{[3]}$. The fields of aesthetic medicine and dentistry have long witnessed the increasing use of autologous blood concentrates, either platelet-rich plasma or the more recent plateletrich fibrin $(\mathrm{PRF})^{[4]}$. Low-speed centrifugation-based PRF, a blood product that has been recently described, appears to have demonstrated additional properties in several in vitro and ex vivo clinical trials ${ }^{[1]}$. PRF can stimulate cell-mediated blood supply and reinforce skin rejuvenation ${ }^{[1]}$. The aim of this literature review is to evaluate the efficacy of microneedling treatment with injectable platelet-rich fibrin (i-PRF) for facial skin rejuvenation applications, using an objective skin analysis system and validated patient-reported outcome measures.

\section{Aging and repair}

Aging is defined as the decline or deterioration of physiologic functions, often attributed to the accumulation of genomic changes, decreased telomere length, protein and cellular damage, increased inflammation and cell senescence, exhaustion of endogenous stem cell populations, and issues with intercellular communication ${ }^{[5]}$. Although not comprehensive, some of the major causes leading to skin aging include ultraviolet damage and other environmental insults, inflammation, and reactive oxidative species that excessively outnumber antioxidant ${ }^{[6,7]}$. Generally, any damage induced by such various sources results in epidermal tissue deterioration and damage as well as to a significant decrease of dermal collagen and elastin levels ${ }^{[8]}$. Aging is also thought to be the cause of decreased epidermal thickness and limited availability of skin growth factors ${ }^{[8]}$. Although they may appear to be distinguishable events, aging and wound healing do exhibit similarities, as they share both genetic and cellular pathways, which compensate and replenish ${ }^{[9]}$.

An additional currently expanding field in terms of skin therapies is the use of growth factors to generate keratinocyte and collagen proliferation. Growth factors are regulatory peptides that engage in cell-to-cell signaling as well as intracellular signaling, such as chemotaxis, division, and differentiation ${ }^{[10]}$. Such proteins can be produced by fibroblasts, platelets, keratinocytes, and immunomodulatory cells. Unlike other peptides known to contribute to intercellular signaling, these proteins are characterized by their role in possessing a targeted response, i.e., a beneficial component in post-wounding skin ${ }^{[11]}$. The specific mechanism of such growth factors involves their diffusion into the wound bed and skin-repair support, while inducing collagen proliferation, promoting angiogenesis, stimulating cell migration and division, and reducing localized inflammation $^{[12]}$. The perception of growth factors in endogenous and exogenous skin aging processes has been clearly demonstrated in clinical trials on skin wound healing ${ }^{[13]}$, and such growth factors have been discovered to repair skin lesions by playing crucial roles in the inflammatory, granulation, and remodeling post-wounding stages observed. In this case, multiple growth factors, such as vascular endothelial growth factor (VEGF), transforming growth factor beta (TGF- $\beta$ ), and interleukin 8, all closely coordinate to 
promote wound resolution ${ }^{[13]}$. Growth factors are deployed in reestablishing the extracellular matrix as well as ensuring sufficient collagen and elastin synthesis, i.e., one of the key goals seen in this phas $\mathrm{e}^{[14]}$. Taking this into account, it can be easily concluded that both the function and mechanism of growth factors in wound healing can be translated in the light of a therapeutic aspect in skin aging, where growth factor count is consumed and skin ultimately ages due to its reduced collagen network ${ }^{[15]}$. In particular, growth factors are able to slow down aging by provoking keratinocytes to deliver larger numbers of growth factors to facilitate collagen production as well as keratinocyte division ${ }^{[8]}$. Such advantages in wound healing enhancement and tissue augmentation are of particular interest in the field of aesthetic medicine, in either surgical or minimally invasive settings, such as microneedling.

\section{Platelet concentrates}

Over the past ten years, the deployment of autologous blood concentrates, such as platelet-rich plasma (PRP) and platelet-rich fibrin (PRF), has proved quite significant and has gained popularity in the field of medical aesthetics, as it can be effectively applied to stimulate, enhance, and rejuvenate the $\operatorname{skin}^{[16-19]}$. Several studies (observational, in vitro, animal models, and clinical trials) suggest a noticeable impact of both topical and injectable applications of platelet concentrates, thusly promoting cellular conversion and facial regeneration ${ }^{[20]}$. This is primarily owed to the fact that the use of autologous platelet growth factors in facial regeneration treatments is considered a natural dermal regeneration restorative approach in contrast to exogenous growth factors and biodegradable compounds. Furthermore, platelet preparations, in addition to their augmenting properties as fillers, deliver multiple growth factors, cytokines, and extracellular matrix proteins as soon as they are activated, i.e., fibrin, fibronectin, and vitronectin ${ }^{[1]}$. These proteins bind to their specific cellular receptors and supplement or alter the diverse intracellular processes that affect cellular reproduction and further extracellular matrix protein output ${ }^{[1,21]}$.

\section{PRP and PRF}

Platelet-rich plasma, thought to represent first-generation platelet concentrates, primarily involves platelets and plasma proteins. PRP preparation entails two-step centrifugation plus the addition of exogenous blood thinners. Moreover, heterologous thrombin or calcium ions are added to PRP to achieve platelet-derived growth factors ${ }^{[20]}$. The use of external chemicals and activation factors may increase the risk of contamination, thus rendering the practical use of PRP a complex procedure mandating studious handling in clinical settings. Over time, the advances achieved in terms of platelet concentrates resulted to the emergence of PRF, a fully autologous, blood-derived biomaterial that is produced via one-step centrifugation and eliminates the need for adding blood thinners ${ }^{[18]}$. Expect for fibrin, platelets, and plasma proteins, PRF involves a great number of white blood cells (WBCs $)^{[20]}$. Determined by the sampling tube and the centrifugation protocol followed, generating either a solid or a liquid PRF matrix without anticoagulants is considered feasible. Regarding solid PRF, platelets interact with the tube's glass surface and trigger their coagulation during centrifugation ${ }^{[18,22]}$. Following the separation process in the centrifuge, the resulting solid PRF matrix consists of a fibrin scaffold with captured platelets, WBCs, plasma proteins, and growth factors. The liquid PRF is generated using a plastic-surface sampling tube to facilitate the production of a liquid PRF matrix in the absence of exogenous blood thinners. The liquid state of the PRF is maintained for ca. 15-20 min, subsequently forming a fibrin $\operatorname{clot}^{[21]}$.

\section{The low-speed centrifugation concept}

Thorough research oriented to the elucidation of the effects centrifugation has on the elements and bioactivity of PRF led to the emergence of the so-called low-speed centrifugation concept (LSCC) ${ }^{[23]}$. According to the concept, the lower adjustment of the relative centrifugation force (RCF) when centrifuging PRF matrices increases platelet and WBC counts in the resultant PRF matrix to a great extent. Moreover, PRF matrices prepared in low RCF produce meaningfully greater concentrations of major growth factors, 
i.e., vascular endothelial growth factor (VEGF), epidermal growth factor (EGF), and platelet-derived growth factor (PDGF-BB), vs. matrices prepared in high RCF settings ${ }^{[21,22,24,25]}$, while they apparently bear a higher regenerative potential over previous platelet preparations ${ }^{[24]}$. A number of PRF matrices can be generated based on the LSCC. The conduction of a systematic evaluation of the RCF impact on cell types and intramatrix production of growth factor ${ }^{[25]}$ led to the description of two major types of matrices generated by two distinct low-speed centrifugation protocols as follows:

(1) Lower RCF protocol: $3 \mathrm{~min}$ at $700 \mathrm{rpm}(60 \mathrm{~g})$.

(2) Higher RCF protocol: 5 min at $1300 \mathrm{rpm}(208 \mathrm{~g})$.

Besides their statistically meaningful differences noted with inflammatory cell and platelet counts as well as the production of several growth factors, such protocols similarly result in PRF matrices of distinctive volumes and mechanical properties ${ }^{[24]}$. More specifically, despite the fact that PRF matrices yielded through higher RCF carry fewer platelets, WBCs, and growth factors, their volumes demonstrate a three-fold increase, as they comprise greater amounts of fibrin and other plasma proteins that are essential to volume measurements (structural support and filling effects) ${ }^{[1]}$.

\section{Preparation of i-PRF}

Prior to any anticipated procedure, a single venous blood sample collected from each patient using a $20 \mathrm{~mL}$ injector was equally divided into two $10 \mathrm{~mL}$ i-PRF tubes; neither tube contained any anticoagulant factor and both were subjected to a 3 min centrifugation at room temperature at $700 \mathrm{rpm}$ ( $60 \mathrm{~g}$ force) with Choukroun PRF Duo Centrifuge (process for PRF, Nice, France) [Figure 1] ${ }^{[26]}$.

\section{Microneedling}

Microneedling causes morphological changes in the skin tissues by inducing direct mechanical traumas. Standard ablation approaches damage soft tissues past the epidermal-dermal junction, efficiently interfering with the basement membrane and initiating fibroblast collagen deposition in a parallel fashion inherent in visible scars ${ }^{[27-30]}$. Percutaneous collagen induction (PCI) is thought to cause more regenerative effects by inducing the normal wound-healing cascade of inflammation, proliferation, and remodeling within normal skin architecture ${ }^{[31-33]}$. When appropriately implemented on the skin, microneedling devices form governeddepth microchannels, whose opening occludes a few minutes later; within this time frame, the professional user of the device delivers topical agents into the microchannels, thus capturing the active substances well into the skin [Figure 2] ${ }^{[34]}$.

Following the controlled penetration of the microneedles into the skin, platelets produce chemotactic factors that facilitate the invasion of other platelets, neutrophils, and fibroblasts ${ }^{[3]}$. The neutrophils are replaced, while the monocytes differentiate into macrophages within the first $48 \mathrm{~h}$. Macrophages release multiple growth factors, including platelet-derived growth factor (PDGF), fibroblast growth factor (FGF), and transforming growth factor (TGF) $-\alpha$ and TGF- $\beta$, which stimulate the migration and proliferation of fibroblasts. In particular, TGF- $\beta 1$, TGF- $\beta 2$, and TGF- $\beta 3$ participate in boosting scar collagen synthesis and regeneration of regular collagen lattice structure and scar-free wound healing ${ }^{[35,36]}$. Keratinocyte-fibroblast interactions actively support the formation of laminin and collagen types IV and VII at the level of an intact basement membrane. In remodeling, i.e., the final phase, fibroblasts keep forming and degrading collagen in the papillary layer of the dermis. Consequently, patients choosing to undergo PCI may well anticipate longterm improvement and increasingly better outcomes in their overall skin quality and density. Over several months, superficial fine lines and wrinkles gradually disappear, unlike ablative laser resurfacing, where the 


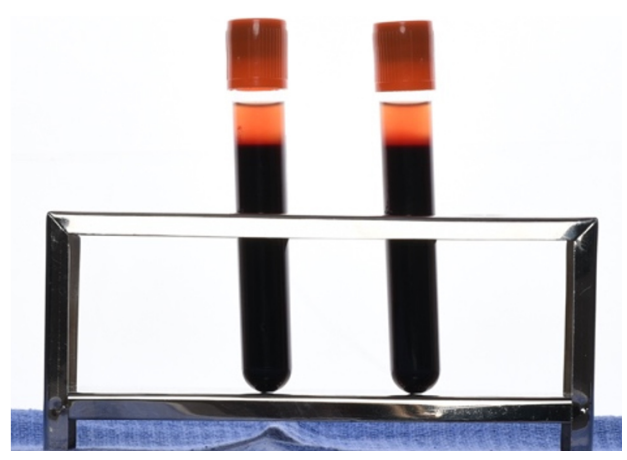

Figure 1. i-PRF right after centrifugation.

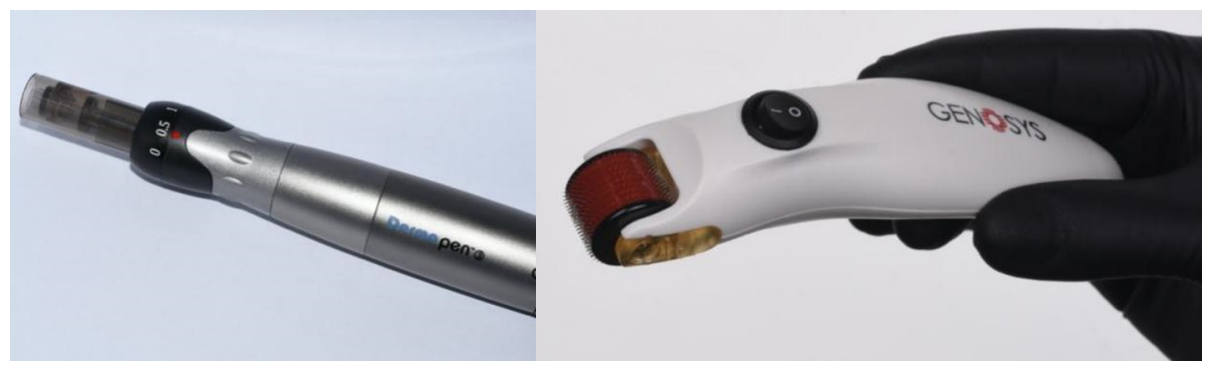

Figure 2. Dermapen and dermaroller.

results achieved are more dramatic and short term.

\section{Clinical benefits}

\section{Painless}

The pain complication accompanying the penetration of needles into the dermis may make patients experience significant discomfort, especially children and people suffering from trypanophobia ${ }^{[37,38]}$. In contrast to hypodermic needles, microneedling treatment entails almost painless needle insertion, thus making this advantage being the most cited one in the literature. The advantage of this mechanism lies within the technical specifications of the needles, i.e., micron size. When the stratum corneum is pierced with microneedles, the mechanoreceptors and nociceptors residing within the dermis are left intact. The pain felt by the patient upon microneedling is proportional to the microneedle length, i.e., the longer is the microneedle, the greater is the possibility to reach and stimulate nociceptors within the viable epidermis ${ }^{[3,40]}$.

\section{Minimally invasive and small lesion size}

Even though standard hypodermic injection is useful for the systemic administration of therapeutic agents, such a modality is perceived as unsophisticated and highly invasive ${ }^{[41]}$. Compared to hypodermic needles, microneedling treatments are characterized by less injection site damage and shorter recovery periods $s^{[42]}$. Furthermore, several in vivo studies, in both humans $s^{[4]}$ and animal models ${ }^{[43]}$, have demonstrated that microneedle penetration is associated with minimal bleeds. A clinical study conducted by Haq et al. ${ }^{[39]}$ (2009), compared the induction of skin puncture between microneedles and hypodermic needles. They found that the lesion induced and the microchannel formed by platinum-coated silicon microneedles demonstrated minimal skin trauma with a more rapid recovery rate ( $8-24 \mathrm{~h})$ compared to that induced by hypodermic needles. Nonetheless, once the microneedles penetrate the stratum corneum (the superficial layer of the epidermis), the risk of infection escalates, regardless of the vascular perfusion in the treated $\operatorname{area}^{[44]}$. However, owed to the skin's self-defense mechanism, multiple studies report that any possible risk 
of infection associated with skin penetration is significantly mitigated by the use of microneedles ${ }^{[45]}$. Therefore, it is evident that the minimally invasive properties of microneedle-assisted drug administration are additional competitive advantages of the device ${ }^{[46]}$.

\section{Equipment}

There is a significant range of equipment available for PCI techniques. Small skin areas and localized scars are well treated with stamp devices that allow spot and focused treatment ${ }^{[40]}$. One of the advantages of electronic stamp devices is that the professional user has the option to select the appropriate settings of penetration speed and needle depth. Thus, patient discomfort is minimized, and the same instrument can be easily and effectively used on various skin areas. In addition, cross-contamination is eliminated, as the stamping device tip cartridges are single-use only. A wide range of needles in terms of material (gold, titanium, etc.), length, diameter, and overall surface density are commercially available for drum-shaped rollers, the technical specifications and individual properties of which vary among manufacturing companies ${ }^{[46]}$. Rollers are single-use devices that best suit open, flat skin surface areas, such as the cheeks. However, it should be noted that they encompass greater difficulties in narrower channels, for example around the mouth and areas adjacent to the scalp, where, occasionally, hair can get captured by the tip of the device and become entrapped in the microneedles ${ }^{[46]}$. Professional-care needles come in a wide range of depths $(0.5-3 \mathrm{~mm})$, but needles for home-care devices, which are intended for self-treatment, are commercially available in depths less than $0.3 \mathrm{~mm}$. In addition to diverse design styles, other features, such as vibration, light-emitting diodes, and radiofrequency, also vary among manufacturing companies. Notwithstanding the type of equipment, an effective and reliable microneedling device is undoubtedly defined by its quality properties; a strong build is crucial for the lowest risk possible of needle breakage into the skin, with ease of use and optimum outcomes being the other two hard requirements for most practitioners $^{[46,47]}$.

\section{Technique}

Patients are strongly advised to avoid prolonged sun exposure or sun burning for at least $24 \mathrm{~h}$ before their treatment session. Thus, the development of excessive inflammation and injury is prevented. The application of topical agents must be ceased $12 \mathrm{~h}$ before the session. The patient's skin must be clean and free from cosmetic products (moisturizer, makeup, etc.). Patients with active or resolving infection must postpone their treatment until all signs and symptoms on the affected area completely resolve. Patient preparation involves cleaning the patient's skin with a mild cleansing product, with or without exfoliation. Next, local anesthesia is applied and left on the skin for $20 \mathrm{~min}$ and is then removed with rubbing alcohol. Local nerve blocks may prove useful and beneficial in skin regions that are sensitive or may demand aggressive treatment, e.g., the upper lip. A lubricant (hyaluronic acid, vitamins, peptides, or hemocomponents) is then layered on the skin, which also has an additional role as an active ingredient that is microinjected into the skin through microneedling action. With regard to the specific device used, practitioners may choose the applicable needle length and penetration speed that best suits the individual therapeutic plan of the area to be treated ${ }^{[47]}$. Thin skin areas that cover bony surfaces, such as on the forehead, on the nose, around the eyes, and on the upper lip, are treated with shorter needles, starting from $0.5 \mathrm{~mm}$. Individuals with higher-density skin or apparent scars may endure greater needle lengths, i.e., 0.5-2 mm. High-density skin areas, such as the face, chest, and trunk, or skin areas with profound scarring can be treated with needle lengths up to $3 \mathrm{~mm}$. Increasing speeds combined with longer needles lessen patient distress and discomfort. Even though needle treatment modalities are user-dependent, a shared endpoint and top priority of all professional users is to restrict any complications that may occur to minimum levels, i.e., mild edema, evenly disseminated erythema, and temporary bleeding on the injection sites. The three pillars to achieve this are: (1) applicable and well-tolerated pressure; (2) speed; and (3) 
needle depth. All of these depend on skin quality and density of the area under treatment. A series of punctures using different vectors on the same area is strongly suggested to prevent the formation of track marks owed to sequential needling into the same microchannel. Track marks inadvertently create larger wounds and unwanted scarring. When the treatment is finished, the patient's skin is thoroughly washed with sterile water to clear any remaining serum and debris. At this stage, the transdermal administration of active components, e.g., topical vitamins, peptides, growth factors, and other substances, can be directly performed, as the patency of the microchannels, although transient, helps their application and absorbance to a great extent ${ }^{[46]}$.

\section{Postprocedural care}

Patients may have a burning sensation, similar to sunburn, and may experience soreness and tenderness. These mild side effects last only for a few hours after the treatment session and can be effectively managed with over-the-counter pain relievers or hyaluronic acid-based moisturizers. The use of sunblock creams/sprays and beauty/skincare products are prohibited for at least $12 \mathrm{~h}$ after the session. As of the next post-treatment day, patients may resume their daily and professional activities; however, their edema may not completely resolve before the second or third post-treatment day. Erythema and mild desquamation may be present until the fifth post-treatment day. After that, routine skincare, including tretinoin, may be resumed. By the end of the first post-treatment week, patients can anticipate no longer experiencing postprocedural adverse reactions. Nevertheless, they should receive strong advice to abstain from alcohol, avoid use acid-based toners, and prevent their skin from direct sun exposure for two weeks. Some patients may present a herpes simplex outbreak, which is effectively prevented with antiviral medication ${ }^{[46,4]}$.

\section{METHODS}

\section{Objective of study}

The aim of this literature review is to evaluate the efficacy of microneedling treatment with i-PRF for facial skin rejuvenation applications, using an objective skin analysis system and validated patient-reported outcome measures, by a single investigator (Vesala AM), under the supervision of the principal investigator (Ruga E).

\section{Design of the study}

This systematic review was performed based on the recommendations and principles of the Cochrane Collaboration as well as the PRISMA statement. Prior to starting this systematic review, an allencompassing protocol was elaborated that received consecutive approval. This highly thorough protocol integrated a number of sections and research techniques, i.e., search approach, determination of eligibility, inclusion requirements, screening methods, data extraction, quality assessment, and data synthesis/analysis. The core question was determined according to the PICO framework: "in all patients undergoing cosmetic surgery $(\mathrm{P})$ with microneedling treatment and the use of i-PRF (I) for face rejuvenation $(\mathrm{O})$ ”.

POPULATION: All patients undergoing cosmetic surgery.

INTERVENTION: Microneedling treatment with injectable platelet rich fibrin (i-PRF).

COMPARISON: Not applicable.

OUTCOME: Face Rejuvenation. 
Inclusion requirements

1. Randomized controlled trials (RCTs), cohort studies, case-control studies, case reports, case series, reviews, editorials, retrospective studies, and single arms of prospective studies.

2. Human studies.

3. Articles written in English.

Exclusion requirements

1. Animal studies.

2. Articles before February 2011.

\section{Search strategy}

The search approach involved the exploration of electronic databases. An advanced search option was applied to filter our search line, i.e., from February 2011 to April 2021. We performed a search on Medline, Scopus, Embase, and Web of Science, while improving the accessed articles via Ovid interface. Our keywords were chiefly aligned with a combination of MeSH terms and text words. Our online search was conducted according to the PICO framework in the following fashion: (microneedling) AND (or) OR (needling) OR (face rejuvenation) OR (skin rejuvenation) OR (facial regeneration) AND (injectable platelet-rich fibrin) OR (i-PRF).

\section{Study eligibility evaluation and data extraction criteria}

The search of the literature was undertaken by a single investigator (Vesala AM), under the supervision of the principal investigator (Ruga E), who is a content expert. Following the exclusion of non-relevant trials, we assessed the eligibility of the remaining publications. A PRISMA flowchart was prepared and used as a standardized screening form, in which we entered all valuable data retrieved from the studies screened at various steps of the review. Following the initial literature search, all article titles were screened again to rule out any non-pertinent publications. As a result, several studies were omitted after viewing and reading data contained in their abstracts. Our closing screening eligibility step involved reading the full text in light of both the inclusion and exclusion requirements.

\section{RESULTS}

The search in the literature yielded 73 studies. After reviewing their title and summary, nine of them were found to meet the inclusion criteria and, next, the full-text articles were reviewed. Of these, three studies were excluded from systematic research, as they would no longer meet the inclusion criteria. In total, six studies were considered for review [Table 1] and the data obtained were summarized on the PRISMA flow chart [Figure 3]. Three studies were excluded from the literature review because of the use of platelet-rich fibrin matrix (PRFM), an older protocol of PRF in aesthetic medicine ${ }^{[48-50]}$.

Wang et al. ${ }^{[24]}$ (2019) investigated the culture of fibroblasts of the dermis, using fluid-PRF and PRP as culture media. Their goal was to study their capacity to stimulate/affect cell viability, migration, spreading, proliferation, and mRNA levels of known mediators of dermal biology including PDGF, TGF- $\beta$, and fibronectin. No platelet concentrate presented toxicity; thus, the cells exhibited high survival rates. The migration rate of skin fibroblasts was above $350 \%$ in the fluid-PRF $v s$. the control and PRP (200\% increase). A boost in cell growth and division was also noted on Day 5, following the induction by fluid-PRF. Whereas both PRP and fluid-PRF generated remarkably high cell mRNA levels of PDGF, the investigators observed markedly higher counts in the TGF- $\beta$, collagen 1, and fibronectin mRNA levels in the fluid-PRF group. 
Table 1. List of articles included in the systematic review

\begin{tabular}{|c|c|c|c|}
\hline Article & Author(s) & Year & Subject \\
\hline $\begin{array}{l}\text { Fluid platelet rich fibrin stimulates greater dermal skin fibroblast cell migration, proliferation, } \\
\text { and collagen synthesis when compared to platelet rich plasma }\end{array}$ & Wang et al. ${ }^{[24]}$ & 2019 & i-PRF vs. PRP \\
\hline $\begin{array}{l}\text { Lower facial regeneration with a combination of platelet rich fibrin liquid matrices based on } \\
\text { the low-speed centrifugation concept Cleopatra technique }\end{array}$ & $\begin{array}{l}\text { Nacopoulos and } \\
\text { Vesala }\end{array}$ & 2019 & $\begin{array}{l}\mathrm{i}-\mathrm{PRF} \text { and skin } \\
\text { rejuvenation }\end{array}$ \\
\hline New frontiers in skin rejuvenation, including stem cells and autologous therapies & Pourang et al. ${ }^{[51]}$ & 2020 & $\begin{array}{l}\mathrm{i}-\mathrm{PRF} \text { and skin } \\
\text { rejuvenation }\end{array}$ \\
\hline Easy platelet-rich fibrin (injectable/topical) for post-resurfacing and microneedle therapy & Gentile $^{[53]}$ & 2020 & $\begin{array}{l}\text { i-PRF and } \\
\text { microneedling }\end{array}$ \\
\hline Injectable platelet-rich fibrin for facial rejuvenation: a prospective, single-center study & Hassan et al. ${ }^{[15]}$ & 2020 & $\begin{array}{l}\mathrm{i}-\mathrm{PRF} \text { and skin } \\
\text { rejuvenation }\end{array}$ \\
\hline $\begin{array}{l}\text { Injectable platelet-rich fibrin (PRF): the newest biomaterial and its use in various } \\
\text { dermatological conditions in our practice: a case series }\end{array}$ & $\begin{array}{l}\text { Shashank and } \\
\text { Bhushan }^{[54]}\end{array}$ & 2020 & $\begin{array}{l}\mathrm{i}-\mathrm{PRF} \text { and skin } \\
\text { rejuvenation }\end{array}$ \\
\hline
\end{tabular}

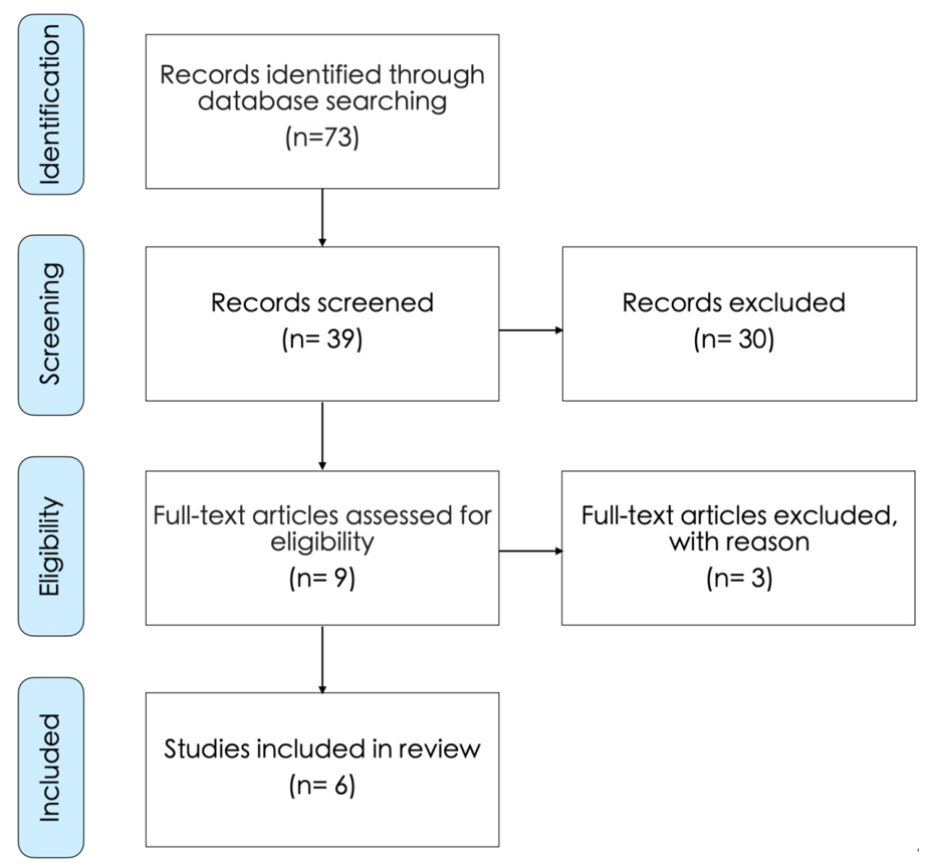

Figure 3. Prisma flow chart.

Finally, fluid-PRF showed a more considerable potential to generate collagen matrix formation versus the PRP. The results from the study of Wang et al. ${ }^{[24]}$ clearly illustrate that the fluid-PRF possesses a more significant regenerative capacity on skin fibroblasts in humans.

Nacopoulos and Vesala ${ }^{[1]}$ (2019) presented a technique (Cleopatra technique) based on the combination of PRF matrices produced by the LSCC for superficial skin rejuvenation and full face rejuvenation. All patients underwent four PRF treatment sessions in total, with each session scheduled 2-3 weeks apart. The first stage of each session involved the collection of $60 \mathrm{~mL}$ of venous blood in $10 \mathrm{~mL}$ PRF tubes (Orange Plastic tubes, Process for PRF, Nice, France). Next, these tubes containing the sampled blood were centrifuged by applying the above-mentioned protocols for low-speed centrifugation. In total, 32 patients underwent the Cleopatra technique with the aim of achieving skin rejuvenation via platelet-rich fibrin. To the best of our knowledge, this is the first published study demonstrating the clinically meaningful advantage of liquid PRF matrices to be used as a single skin rejuvenation treatment modality ${ }^{[1]}$. 
Pourang et al. ${ }^{[51]}$ (2020) demonstrated that the localized delivery of growth factors can enhance human skin, in terms of both texture and appearance. Localized delivery of PRF during and right after microneedling treatment may produce better outcomes through saturation of the newly shaped fine, porous wounds with concentrated growth factors. Naturally and physiologically activated PRF, combined with its extended delivery of growth factors, maintained the signals of healing and regeneration with a longer duration. Along with this study, PRF demonstrated its ability to produce higher overall growth factor concentrations when compared to $\mathrm{PRP}{ }^{[52]}$.

Gentile $^{[53]}$ (2020) showed that PRF comprises cytokines, polysaccharide chains, and structural glycoproteins, all forming parts of an autologous fibrin meshwork that polymerizes in a slow fashion. Three main growth factors are largely released by the PRF, i.e., TGF $\beta 1$, platelet-derived GF-AB, and vascular endothelial GF. In addition, the seven-day production of a significant coagulation matricellular glycoprotein (thrombospondin-1) is observed. Platelet biologics are made of major growth factors that enhance cell growth and division, collagen production, chemotaxis, angiogenesis, and cell differentiation ${ }^{[53]}$.

Hassan et al. ${ }^{[15]}$ (2020), in a single-center, prospective, uncontrolled study assessed the efficacy of i-PRF for skin regeneration through an unbiased skin analysis system and validated patient-reported outcome measures (PROMs). Eleven healthy female participants were enrolled in the study; over a three-month period, the subjects received intradermal i-PRF injections in three areas of their face on a monthly basis: malar areas ( $1 \mathrm{~mL}$ each side), nasolabial fold ( $0.5 \mathrm{~mL}$ each side), and upper lip skin above the vermilion border $(1 \mathrm{~mL})$. The outcomes of the treatments on the subjects' skin were measured via the VISIA ${ }^{\star}$ Complexion Analysis System. In addition, the outcomes were estimated based on the self-questionnaire for patient-reported outcomes (FACE-Q), which was handed out at baseline and after Month 3. At their threemonth follow-up visit, patients had significant skin amelioration at both spot $(P=0.01)$ and pore levels $(P=$ 0.03). It should be noted that other study components also exhibited numerical increase, such as skin texture, wrinkles, ultraviolet spots, and porphyrins. All components of the FACE-Q questionnaire for the measurement of patient satisfaction regarding the item of appearance presented an important improvement compared to the initial skin assessment, such as satisfaction with skin $(P=0.002)$, satisfaction with facial appearance $(P=0.025)$, satisfaction with cheeks $(P=0.001)$, satisfaction with lower face and jawline $(P=$ $0.002)$, and satisfaction with lips $(P=0.04)$. There was no report of significant adverse reactions by any participant. A series of three i-PRF injections led to remarkable skin rejuvenation that was evident at the three-month follow-up visit, as demonstrated by the skin analysis components that were clearly bettered and the scores measured on the patient self-questionnaires ${ }^{[15]}$.

Shashank and Bhushan ${ }^{[54]}$ (2020) performed a case series study and discovered that injectable PRF (i-PRF) is able to rejuvenate the area around the eyes, given it gradually and steadily releases growth factors in the long run. A 39-year-old female patient presented with complaints of having a "tired look" due to dull-looking infraorbital skin, combined with fine line and wrinkles and blackish-brown infraorbital blemishes. The treatment plan for this patient comprised three treatment sessions, with each session scheduled one month apart. Following each injection, there was a directly evident filling of the infraorbital hollow, which was sustained for approximately 15 days. On completion of the third session, the treated patient self-assessed the outcomes and reported decreased skin laxity, skin texture improvement, discoloration reduction, and a fuller infraorbital hollow, which gave her a rejuvenated appearance ${ }^{[54]}$.

\section{DISCUSSION}

Facial aesthetics is increasingly performed in the majority of countries. This reflects a trend toward more conservative procedures or alternatives to surgical restoration. The rationale behind it is that the 
enhancement of facial features, the correction of facial asymmetry, and the restoration of age-induced facial volume loss cannot be effectively achieved with the conventional excision and suspension procedures ${ }^{[1]}$. Microneedling treatments are gaining increasing acceptance and demand for the management of a wide spectrum of skin conditions ${ }^{[55]}$, mostly including atrophic acne scarring ${ }^{[56]}$, stretch marks $s^{[57]}$, melasmas $s^{[58]}$, and skin regeneration ${ }^{[59]}$. Microneedling treatments for the skin are also used along with transplanting skin cells to treat patients with burn injuries as well as for the transdermal administration of agents ${ }^{[0-62]}$. In addition, microneedling treatments are not only affordable but also easy to handle $v s$. ablative and nonablative laser treatment modalities; thus, this approach is rendered more and more popular, while it promotes research in this field in unprecedented rates ${ }^{[2,63]}$. Not all dermal therapies are identical - from chemical methods to laser therapies and surgical procedures, practitioners may select the skin treatment modality that best fits their patient's individual needs ${ }^{[31]}$. Nevertheless, the majority of such therapies are invasive and may induce secondary skin issues such as hyper- or hypo-pigmentation, particularly in darkskinned patients ${ }^{[64]}$. Study findings on microneedling (1-1.5 mm needle length) outcomes are strongly favorable for skin rejuvenating purposes, while they present great similarities to results obtained from medical needling ( $3 \mathrm{~mm}$ needle length ${ }^{[2]}$. Being less invasive, microneedling has yet another advantage, as it only requires local anesthesia administration. Moreover, potential post-treatment complications, such as side effects, bleeds, edema, and pain, are minimized, because the epidermis remains almost unharmed ${ }^{[65]}$. To date, there is no expanded knowledge on the underlying molecular and histomorphological impact of microneedling procedures on human skin. The reason is that any observed expressional changes of several growth factors (TGF $\beta 1-3$, FGF, EGF, VEGF, and TNF- $\alpha$ ) that induce collagen formation have only been reported in animal studies ${ }^{[61,64]}$.

Natural filling materials, such as collagen, hyaluronic acid, fat, PRP, and PRF, constitute a rational and potentially successful treatment option for this purpose, whether alone or supplementary to surgical facial rejuvenation approaches. Over time, practitioners have investigated the potential of retrieving soft tissue augmentation material from autologous sources, particularly in the field of aesthetic medicine. As foreign materials, due to their biodegradable properties, may potentially cause transient effects, a major unmet patient need is undoubtedly identified that includes the prevention of both granuloma formation and chronic or late-onset infection development ${ }^{[24]}$. Additionally, the safety of autologous growth factors originating outside an organism, primarily in correlation to cancer, has not been established. As a consequence, autologous platelet products comprising growth factors, e.g., PDGF, TGF, particularly TGF- $\beta$, VEGF, insulin-like growth factor, and EGF, have been studied to a great extent over the last ten years ${ }^{[6 \sigma]}$. Besides their direct augmenting properties, platelet concentrates boost angiogenesis and enhance collagen and fibronectin production ${ }^{[6]}$. Platelet concentrates have demonstrated that their chemotactic and/or mitogenic activity in a number of cell types, including monocytes, fibroblasts, stem cells, smooth muscle cells, endothelial cells, and keratinocytes, is maintained ${ }^{[68]}$.

Stem cell-based therapies have been widely used for their abilities to repair and regenerate different types of tissues and organs in cosmetic and plastic surgeries. It involves the clinical application of different types of stem cells. Different stem cells have been reported to be applicable in different areas of cosmetic surgeries such as face lipoatrophy, skin rejuvenation, breast enhancement, and body contouring. However, adiposederived stem cells remains the most widely used by cosmetic surgeons as they have the potential and capability to differentiate into mesenchymal, ectodermal, and endodermal lineages and are easily accessible to harvest ${ }^{[69]}$.

Aesthetic surgery-based experiences have shown that transferring autologous fat is helpful and will aid in short-acting fillers ${ }^{[0]}$. This evolutionary procedure is one of the most commonly used strategies for aesthetic 
and reconstructive operations, including soft-tissue defects, facial rejuvenation, and breast augmentation, because of its multiple advantages (e.g., abundance, ease of collection, formation of microlesions, and lack of allergic reactions $)^{[7]}$. Recent studies have shown that early neovascularization of grafted fat plays a critical role in improving the quality and retention of transplanted tissue ${ }^{[72-74]}$. To augment the retained percentage of fat grafts reducing the percentage of necrosis, angiogenesis is needed for nutrition and incorporation within the surrounding tissue. Cell-assisted lipotransfer can lead to a fall in postoperative atrophy and augment neovascularization ${ }^{[70]}$.

Platelet concentrates, concentrated solutions of autologous platelets prepared by collecting the patient's own blood and submitting it to centrifugation several times, may help to address this predicament in fat grafting.

Platelet-rich plasma, a first-generation platelet concentrate, contributes to the degranulation of platelets, resulting in the release of various growth factors, including VEGF, basic FGF, PDGF, and epithelial growth factor-and cytokines ${ }^{[75]}$. Platelet-rich plasma reportedly improves grafted fat retention ${ }^{[76,77]}$ by means of increased neovascularization ${ }^{[78]}$, enhanced proliferation and differentiation of adipose-derived stem cells ${ }^{[79]}$, and direct nutrient infiltration to the grafts. However, using exogenous additives during platelet-rich plasma preparation may cause adverse effects. Moreover, applying activators can trigger the sudden release of growth factors in platelet-rich plasma within one day, which may reduce its efficacy ${ }^{[80]}$. Importantly, the efficacy of platelet-rich plasma for outcomes in fat grafting remains controversial ${ }^{[81]}$.

The second-generation platelet concentrate platelet-rich fibrin was recently reported ${ }^{[82]}$; it is superior to platelet-rich plasma in many aspects, with its preparation being simpler than that of platelet-rich plasma and requiring only one centrifugation step. Moreover, it does not require the addition of exogenous additives, which promote natural physiologic polymerization of fibrin. The three-dimensional fibrin structure of platelet-rich fibrin facilitates platelet capture and growth factor bonding, which enhances the gradual and long-tern release of growth factors and cytokines ${ }^{[83,84]}$. This fibrin mesh also provides a framework for cell proliferation and differentiation and new blood vessel formation.

A clinical self-control study comparing the effects of platelet-rich fibrin or platelet-rich plasma combined with a fat graft in facial lipostructure indicated that a greater average resorption was observed on the platelet-rich plasma/fat side $(0.9 \pm 0.3$ with platelet-rich fibrin $v s .1 .4 \pm 0.5$ with platelet-rich plasma). However, the results are subjective and inconclusive, as the study evaluated the tissue resorption by comparing presurgical and postsurgical photographic views rather than using a more objective measurement such as three-dimensional scanning or magnetic resonance imaging ${ }^{[85]}$. Thus, platelet-rich fibrin may perform better and replace platelet-rich plasma in fat-grafting applications, although further studies are needed to support this. This study compared the effects of platelet-rich plasma or platelet-rich fibrin associated with fat grafting by histologically evaluating their functions in a rabbit fat transplantation $\operatorname{model}^{[80]}$.

In addition, an in vivo study found that optimized LSCC-prepared PRF matrices perfuse blood vessels at higher rates than PRF matrices prepared through high centrifugation force ${ }^{[86]}$, while they seem to have a more considerable regenerative potential compared to earlier platelets preparations ${ }^{[24]}$. On that account, liquid PRF may well constitute a growth factor material of autologous origin that can stimulate cellmediated blood supply and reinforce skin rejuvenation. Furthermore, genetic factors could have a potential use as biomarkers or predictors for skin rejuvenation PRF protocols in the application of medical aesthetics on the face $e^{[1,87]}$. 


\section{Conclusion}

Further research on PRP and PRF is warranted to better elucidate their functional roles in medical cosmetic rejuvenation. Although PRP has a more extensive history of applied use, research on the functionality and sustainability of growth factors and other regenerative cells in purely autologous PRF justifies its continued use. Comparative studies including both treatments may provide additional insight into the preferential implications of each.

\section{DECLARATIONS}

\section{Authors' contributions}

Wrote the manuscript: Vesala AM, Ruga E

Conducted the reaserach of the literature: Vesala AM, Ruga E, Nacopoulos C, Gkouskou K

Designed the study: Vesala AM, Ruga E, Amenta F

This work was carried out in collaboration between all authors.

All authors read and approved the final manuscript.

\section{Availability of data and materials}

Not applicable.

\section{Financial support and sponsorship}

This work was supported by an institutional grant of the University of Camerino.

\section{Conflicts of interest}

All authors declared that there are no conflicts of interest.

\section{Ethical approval and consent to participate}

Not applicable.

\section{Consent for publication}

All figures obtained copyright permission.

\section{Copyright}

(C) The Author(s) 2021.

\section{REFERENCES}

1. Nacopoulos C, Vesala AM. Lower facial regeneration with a combination of platelet-rich fibrin liquid matrices based on the low speed centrifugation concept-Cleopatra technique. J Cosmet Dermatol 2020;19:185-9. DOI PubMed

2. Hou A, Cohen B, Haimovic A, Elbuluk N. Microneedling: a comprehensive review. Dermatol Surg 2017;43:321-39. DOI PubMed

3. Amer M, Farag F, Amer A, ElKot R, Mahmoud R. Dermapen in the treatment of wrinkles in cigarette smokers and skin aging effectively. J Cosmet Dermatol 2018;17:1200-4. DOI PubMed

4. Miron RJ, Zucchelli G, Pikos MA, et al. Use of platelet-rich fibrin in regenerative dentistry: a systematic review. Clin Oral Investig 2017;21:1913-27. DOI PubMed

5. López-Otín C, Blasco MA, Partridge L, Serrano M, Kroemer G. The hallmarks of aging. Cell 2013;153:1194-217. DOI PubMed PMC

6. Quan T, He T, Kang S, Voorhees JJ, Fisher GJ. Solar ultraviolet irradiation reduces collagen in photoaged human skin by blocking transforming growth factor- $\beta$ type II receptor/Smad signaling. Am J Pathol 2004;165:741-51. DOI PubMed PMC

7. Yamamoto Y, Gaynor RB. Therapeutic potential of inhibition of the NF-kappaB pathway in the treatment of inflammation and cancer. J Clin Invest 2001;107:135-42. DOI PubMed PMC

8. Fabi S, Sundaram H. The potential of topical and injectable growth factors and cytokines for skin rejuvenation. Facial Plast Surg 2014;30:157-71. DOI PubMed

9. Taub AF, Pham K. Stem cells in dermatology and anti-aging care of the skin. Facial Plast Surg Clin North Am 2018;26:425-37. DOI PubMed

10. Mehta RC, Fitzpatrick RE. Endogenous growth factors as cosmeceuticals. Dermatol Ther 2007;20:350-9. DOI PubMed

11. Varela HA, Souza JCM, Nascimento RM, et al. Injectable platelet rich fibrin: cell content, morphological, and protein characterization. 
Clin Oral Investig 2019;23:1309-18. DOI PubMed

12. Sundaram H, Mehta RC, Norine JA, et al. Topically applied physiologically balanced growth factors: a new paradigm of skin rejuvenation. J Drugs Dermatol 2009;8:4-13. PubMed

13. Moulin V. Growth factors in skin wound healing. Eur J Cell Biol 1995;68:1-7. PubMed

14. Cheng M, Wang H, Yoshida R, Murray MM. Platelets and plasma proteins are both required to stimulate collagen gene expression by anterior cruciate ligament cells in three-dimensional culture. Tissue Eng Part A 2010;16:1479-89. DOI PubMed PMC

15. Hassan H, Quinlan DJ, Ghanem A. Injectable platelet-rich fibrin for facial rejuvenation: a prospective, single-center study. J Cosmet Dermatol 2020;19:3213-21. DOI PubMed

16. Kim DH, Je YJ, Kim CD, et al. Can platelet-rich plasma be used for skin rejuvenation? Ann Dermatol 2011;23:424-31. DOI PubMed PMC

17. Redaelli A, Romano D, Marciano A. Face and neck revitalization with platelet-rich plasma (PRP): clinical outcome in a series of 23 consecutively treated patients. J Drugs Dermatol 2010;9:466-72. PubMed

18. Sclafani AP. Applications of platelet-rich fibrin matrix in facial plastic surgery. Facial Plast Surg 2009;25:270-6. DOI PubMed

19. Sclafani AP. Platelet-rich fibrin matrix for improvement of deep nasolabial folds. J Cosmet Dermatol 2010;9:66-71. DOI PubMed

20. Elghblawi E. Platelet-rich plasma, the ultimate secret for youthful skin elixir and hair growth triggering. J Cosmet Dermatol 2018;17:423-30. DOI PubMed

21. Martínez-Zapata MJ, Martí-Carvajal A, Solà I, et al. Efficacy and safety of the use of autologous plasma rich in platelets for tissue regeneration: a systematic review. Transfusion 2009;49:44-56. DOI PubMed

22. Donofrio LM. Fat distribution: a morphologic study of the aging face. Dermatol Surg 2000;26:1107-12. PubMed

23. Choukroun J, Ghanaati S. Reduction of relative centrifugation force within injectable platelet-rich-fibrin (PRF) concentrates advances patients' own inflammatory cells, platelets and growth factors: the first introduction to the low speed centrifugation concept. Eur $J$ Trauma Emerg Surg 2018;44:87-95. DOI PubMed PMC

24. Wang X, Yang Y, Zhang Y, Miron RJ. Fluid platelet-rich fibrin stimulates greater dermal skin fibroblast cell migration, proliferation, and collagen synthesis when compared to platelet-rich plasma. J Cosmet Dermatol 2019;18:2004-10. DOI PubMed

25. Wend S, Kubesch A, Orlowska A, et al. Reduction of the relative centrifugal force influences cell number and growth factor release within injectable PRF-based matrices. J Mater Sci Mater Med 2017;28:188. DOI PubMed

26. Ozsagir ZB, Saglam E, Sen Yilmaz B, Choukroun J, Tunali M. Injectable platelet-rich fibrin and microneedling for gingival augmentation in thin periodontal phenotype: a randomized controlled clinical trial. J Clin Periodontol 2020;47:489-99. DOI PubMed

27. Roy D. Ablative facial resurfacing. Dermatol Clin 2005;23:549-59, viii. DOI PubMed

28. Ross E, Naseef GS, Mckinlay JR, et al. Comparison of carbon dioxide laser, erbium:YAG laser, dermabrasion, and dermatome. $J$ Am Acad Dermatol 2000;42:92-105. DOI PubMed

29. Bernstein LJ, Kauvar AN, Grossman MC, Geronemus RG. The short- and long-term side effects of carbon dioxide laser resurfacing. Dermatol Surg 1997;23:519-25. DOI PubMed

30. Laws RA, Finley EM, McCollough ML, Grabski WJ. Alabaster skin after carbon dioxide laser resurfacing with histologic correlation. Dermatol Surg 1998;24:633-6. DOI PubMed

31. Fabbrocini G, Fardella N, Monfrecola A, Proietti I, Innocenzi D. Acne scarring treatment using skin needling. Clin Exp Dermatol 2009;34:874-9. DOI PubMed

32. Fife D. Practical evaluation and management of atrophic acne scars: tips for the general dermatologist. $J$ Clin Aesthet Dermatol 2011;4:50-7. PubMed PMC

33. El-Domyati M, Barakat M, Awad S, Medhat W, El-Fakahany H, Farag H. Microneedling therapy for atrophic acne scars: an objective evaluation. J Clin Aesthet Dermatol 2015;8:36-42. PubMed PMC

34. Schwarz M, Laaff H. A prospective controlled assessment of microneedling with the Dermaroller device. Plast Reconstr Surg 2011;127:146e-8e. DOI PubMed

35. Ferguson MW, O'Kane S. Scar-free healing: from embryonic mechanisms to adult therapeutic intervention. Philos Trans R Soc Lond B Biol Sci 2004;359:839-50. DOI PubMed PMC

36. Bandyopadhyay B, Fan J, Guan S, et al. A "traffic control" role for TGFbeta3: orchestrating dermal and epidermal cell motility during wound healing. J Cell Biol 2006;172:1093-105. DOI PubMed PMC

37. Nir Y, Paz A, Sabo E, Potasman I. Fear of injections in young adults: prevalence and associations. Am J Trop Med Hyg 2003;68:341-4. PubMed

38. Hamilton JG. Needle phobia: a neglected diagnosis. J Fam Pract 1995;41:169-75. PubMed

39. Haq MI, Smith E, John DN, et al. Clinical administration of microneedles: skin puncture, pain and sensation. Biomed Microdevices 2009;11:35-47. DOI PubMed

40. Dsouza L, Ghate VM, Lewis SA. Derma rollers in therapy: the transition from cosmetics to transdermal drug delivery. Biomed Microdevices 2020;22:77. DOI PubMed PMC

41. Li WZ, Huo MR, Zhou JP, et al. Super-short solid silicon microneedles for transdermal drug delivery applications. Int $J$ Pharm 2010;389:122-9. DOI PubMed

42. Bariya SH, Gohel MC, Mehta TA, Sharma OP. Microneedles: an emerging transdermal drug delivery system. $J$ Pharm Pharmacol 2012;64:11-29. DOI PubMed

43. Matriano JA, Cormier M, Johnson J, et al. Macroflux microprojection array patch technology: a new and efficient approach for intracutaneous immunization. Pharm Res 2002;19:63-70. DOI PubMed

44. Donnelly RF, Singh TR, Garland MJ, et al. Hydrogel-forming microneedle arrays for enhanced transdermal drug delivery. Adv Funct 
Mater 2012;22:4879-90. DOI PubMed PMC

45. Kim YC, Park JH, Prausnitz MR. Microneedles for drug and vaccine delivery. Adv Drug Deliv Rev 2012;64:1547-68. DOI PubMed PMC

46. Sabri AH, Ogilvie J, Abdulhamid K, et al. Expanding the applications of microneedles in dermatology. Eur J Pharm Biopharm 2019;140:121-40. DOI PubMed

47. Lee JC, Daniels MA, Roth MZ. Mesotherapy, microneedling, and chemical peels. Clin Plast Surg 2016;43:583-95. DOI PubMed

48. Sclafani AP, McCormick SA. Induction of dermal collagenesis, angiogenesis, and adipogenesis in human skin by injection of plateletrich fibrin matrix. Arch Facial Plast Surg 2012;14:132-6. DOI PubMed

49. Sclafani AP, Saman M. Platelet-rich fibrin matrix for facial plastic surgery. Facial Plast Surg Clin North Am 2012;20:177-86, vi. DOI PubMed

50. Sclafani AP. Safety, efficacy, and utility of platelet-rich fibrin matrix in facial plastic surgery. Arch Facial Plast Surg 2011;13:247-51. DOI PubMed

51. Pourang A, Rockwell H, Karimi K. New frontiers in skin rejuvenation, including stem cells and autologous therapies. Facial Plast Surg Clin North Am 2020;28:101-17. DOI PubMed

52. Masuki H, Okudera T, Watanebe T, et al. Growth factor and pro-inflammatory cytokine contents in platelet-rich plasma (PRP), plasma rich in growth factors (PRGF), advanced platelet-rich fibrin (A-PRF), and concentrated growth factors (CGF). Int J Implant Dent 2016;2:19. DOI PubMed PMC

53. Gentile RD. Easy platelet-rich fibrin (injectable/topical) for post-resurfacing and microneedle therapy. Facial Plast Surg Clin North Am 2020;28:127-34. DOI PubMed

54. Shashank B, Bhushan M. Injectable platelet-rich fibrin (PRF): the newest biomaterial and its use in various dermatological conditions in our practice: a case series. J Cosmet Dermatol 2021;20:1421-6. DOI PubMed

55. Bonati LM, Epstein GK, Strugar TL. Microneedling in all skin types: a review. J Drugs Dermatol 2017;16:308-13. PubMed

56. Cachafeiro T, Escobar G, Maldonado G, Cestari T, Corleta O. Comparison of nonablative fractional erbium laser 1,340 nm and microneedling for the treatment of atrophic acne scars: a randomized clinical trial. Dermatol Surg 2016;42:232-41. DOI PubMed

57. Nassar A, Ghomey S, El Gohary Y, El-Desoky F. Treatment of striae distensae with needling therapy versus microdermabrasion with sonophoresis. J Cosmet Laser Ther 2016;18:330-4. DOI PubMed

58. Ede A. Microneedling in facial recalcitrant melasma: report of a series of 22 cases. An Bras Dermatol 2015;90:919-21. DOI PubMed PMC

59. Singh A, Yadav S. Microneedling: advances and widening horizons. Indian Dermatol Online J 2016;7:244-54. DOI PubMed PMC

60. Busch KH, Bender R, Walezko N, Aziz H, Altintas MA, Aust MC. Combination of medical needling and non-cultured autologous skin cell transplantation (renovacell) for repigmentation of hypopigmented burn scars in children and young people. Ann Burns Fire Disasters 2016;29:116-22. PubMed PMC

61. Zeitter S, Sikora Z, Jahn S, et al. Microneedling: matching the results of medical needling and repetitive treatments to maximize potential for skin regeneration. Burns 2014;40:966-73. DOI PubMed

62. Spencer JM, Freeman SA. Microneedling prior to levulan PDT for the treatment of actinic keratoses: a split-face, blinded trial. $J$ Drugs Dermatol 2016;15:1072-4. PubMed

63. Rivera AE. Acne scarring: a review and current treatment modalities. J Am Acad Dermatol 2008;59:659-76. DOI PubMed

64. Schmitt L, Marquardt Y, Amann P, et al. Comprehensive molecular characterization of microneedling therapy in a human threedimensional skin model. PLoS One 2018;13:e204318. DOI PubMed PMC

65. Fernandes D, Signorini M. Combating photoaging with percutaneous collagen induction. Clin Dermatol 2008;26:192-9. DOI PubMed

66. Choi SW, Pangeni R, Jung DH, Kim SJ, Park JW. Construction and characterization of cell-penetrating peptide-fused fibroblast growth factor and vascular endothelial growth factor for an enhanced percutaneous delivery system. J Nanosci Nanotechnol 2018;18:842-7. DOI PubMed

67. Wang X, Zhang Y, Choukroun J, Ghanaati S, Miron RJ. Behavior of gingival fibroblasts on titanium implant surfaces in combination with either injectable-PRF or PRP. Int J Mol Sci 2017;18:331. DOI PubMed PMC

68. Miron RJ, Fujioka-Kobayashi M, Bishara M, Zhang Y, Hernandez M, Choukroun J. Platelet-rich fibrin and soft tissue wound healing: a systematic review. Tissue Eng Part B Rev 2017;23:83-99. DOI PubMed

69. Zarei F, Abbaszadeh A. Stem cell and skin rejuvenation. J Cosmet Laser Ther 2018;20:193-7. DOI PubMed

70. Gentile P, Calabrese C, De Angelis B, Pizzicannella J, Kothari A, Garcovich S. Impact of the different preparation methods to obtain human adipose-derived stromal vascular fraction cells (AD-SVFs) and human adipose-derived mesenchymal stem cells (AD-MSCs): enzymatic digestion versus mechanical centrifugation. Int J Mol Sci 2019;20:5471. DOI PubMed PMC

71. Pu LLQ. Fat grafting for facial rejuvenation and contouring: a rationalized approach. Ann Plast Surg 2018;81:S102-8. DOI PubMed

72. Pu LL. Mechanisms of fat graft survival. Ann Plast Surg 2016;77 Suppl 1:S84-6. DOI PubMed

73. Khouri RK Jr. Discussion: improved long-term volume retention of stromal vascular fraction gel grafting with enhanced angiogenesis and adipogenesis. Plast Reconstr Surg 2018;141:687e-8e. DOI PubMed

74. Zhang Y, Cai J, Zhou T, Yao Y, Dong Z, Lu F. Improved long-term volume retention of stromal vascular fraction gel grafting with enhanced angiogenesis and adipogenesis. Plast Reconstr Surg 2018;141:676e-86e. DOI PubMed

75. Frautschi RS, Hashem AM, Halasa B, Cakmakoglu C, Zins JE. Current evidence for clinical efficacy of platelet rich plasma in aesthetic surgery: a systematic review. Aesthet Surg J 2017;37:353-62. DOI PubMed

76. Cervelli V, Palla L, Pascali M, De Angelis B, Curcio BC, Gentile P. Autologous platelet-rich plasma mixed with purified fat graft in 
aesthetic plastic surgery. Aesthetic Plast Surg 2009;33:716-21. DOI PubMed

77. Sasaki GH. The Safety and efficacy of cell-assisted fat grafting to traditional fat grafting in the anterior mid-face: an indirect assessment by 3D imaging. Aesthetic Plast Surg 2015;39:833-46. DOI PubMed

78. Mammoto T, Jiang A, Jiang E, Mammoto A. Platelet rich plasma extract promotes angiogenesis through the angiopoietin1-Tie2 pathway. Microvasc Res 2013;89:15-24. DOI PubMed

79. Felthaus O, Prantl L, Skaff-Schwarze M, et al. Effects of different concentrations of platelet-rich plasma and platelet-poor plasma on vitality and differentiation of autologous adipose tissue-derived stem cells. Clin Hemorheol Microcirc 2017;66:47-55. DOI PubMed

80. Xiong S, Qiu L, Su Y, Zheng H, Yi C. Platelet-rich plasma and platelet-rich fibrin enhance the outcomes of fat grafting: a comparative study. Plast Reconstr Surg 2019;143:1201e-12e. DOI PubMed

81. Luck J, Smith OJ, Mosahebi A. A systematic review of autologous platelet-rich plasma and fat graft preparation methods. Plast Reconstr Surg Glob Open 2017;5:e1596. DOI PubMed PMC

82. Dohan DM, Choukroun J, Diss A, et al. Platelet-rich fibrin (PRF): a second-generation platelet concentrate. Part II: platelet-related biologic features. Oral Surg Oral Med Oral Pathol Oral Radiol Endod 2006;101:e45-50. DOI PubMed

83. Dohan DM, Choukroun J, Diss A, et al. Platelet-rich fibrin (PRF): a second-generation platelet concentrate. Part III: leucocyte activation: a new feature for platelet concentrates? Oral Surg Oral Med Oral Pathol Oral Radiol Endod 2006;101:e51-5. DOI PubMed

84. Jiménez-Aristizabal RF, López C, Álvarez ME, Giraldo C, Prades M, Carmona JU. Long-term cytokine and growth factor release from equine platelet-rich fibrin clots obtained with two different centrifugation protocols. Cytokine 2017;97:149-55. DOI PubMed

85. Keyhan SO, Hemmat S, Badri AA, Abdeshahzadeh A, Khiabani K. Use of platelet-rich fibrin and platelet-rich plasma in combination with fat graft: which is more effective during facial lipostructure? J Oral Maxillofac Surg 2013;71:610-21. DOI PubMed

86. Kubesch A, Barbeck M, Al-Maawi S, et al. A low-speed centrifugation concept leads to cell accumulation and vascularization of solid platelet-rich fibrin: an experimental study in vivo. Platelets 2019;30:329-40. DOI PubMed

87. Nacopoulos C, Gkouskou K, Karypidis D, et al. Telomere length and genetic variations affecting telomere length as biomarkers for facial regeneration with platelet-rich fibrin based on the low-speed centrifugation concept. J Cosmet Dermatol 2019;18:408-13. DOI PubMed 